\title{
Desarrollo de nuevas competencias con el método de proyectos en la formación profesional universitaria Development of new competences with the project method in university professional training
}

\author{
Julio Ernesto Quispe Rojas ${ }^{1}$, Julio Enrique Quispe Tuesta ${ }^{2}$ \\ jquisperojas@hotmail.com,julioqt@gmail.com \\ ${ }^{1}$ Departamento de Computación y Electrónica \\ Universidad Nacional Pedro Ruiz Gallo \\ Lambayeque, Perú \\ ${ }^{2}$ Escuela de Ingeniería de Sistemas \\ Universidad Nacional Tecnológica de Lima Sur \\ Lima, Perú
}

\begin{abstract}
Resumen- La formación universitaria tradicional prioriza la ponencia didáctica para los conocimientos, las prácticas de laboratorio para las habilidades; la motivación y recomendaciones para mejorar las actitudes; en cambio el método de proyectos de enseñanza aprendizaje realiza una acción completa a cargo del estudiante, desde la búsqueda de información, planificación, decisión, ejecución, control y valoración, y el docente tiene la función de facilitador y arbitro para el reto y competencia leal entre los equipos de trabajo que llevan el curso con aplicación del método de proyectos. El presente trabajo comparte las experiencias de aplicación en el curso de circuitos de radiocomunicación de la carrera de ingeniería electrónica en la Universidad Nacional Pedro Ruiz Gallo, en los dos ciclos del año 2016, con la finalidad de desarrollar las competencias de trabajo en equipo, investigación y aprendizaje autónomo, toma de decisiones, liderazgo y tolerancia, solidaridad e identificación de grupo. Y también con la finalidad de evidenciar que los alumnos que tienen un perfil bajo en los cursos con el método tradicional, suelen destacar en los cursos en los que se aplica la acción y la autonomía en el aprendizaje, como el método de proyectos.
\end{abstract}

Palabras clave: método de proyectos, desarrollo de competencias, formación profesional universitaria.

Abstract- The traditional university training prioritizes the didactic presentation for the knowledge, the laboratory practices for the skills; Motivation and recommendations to improve attitudes; On the other hand, the method of learning teaching projects involves student work, information search, planning, decision making, execution, control and assessment, and the teacher has the role of facilitator and Arbitration for the challenge and fair competition Among the work teams that take the course with the use of the project method. The present work compares the experiences of the application in the course of radio communication circuits of the electronic engineering career at the National University Pedro Ruiz Gallo, in the two cycles of the year 2016, with the purpose of developing the skills of teamwork, research And autonomous learning, decision making, leadership and tolerance, solidarity and group identification. And also with the purpose of evidencing that students who have a low profile in the courses with the traditional method, usually emphasize in the courses in which the action is applied and the autonomy in the learning, method of projects.

Keywords: project method, competence development, university vocational training.

\section{INTRODUCCIÓN}

La formación universitaria tradicional en la Universidad Nacional Pedro Ruiz Gallo - UNPRG, esta basada en las ponencias didácticas para la teoría, quizás un control de estudio previo al iniciar la clase sobre el tema a tratar y una clase activa con amplia participación de los estudiantes; practicas de laboratorio para logro de las habilidades, con guías de prácticas para orientar y a veces una demostración; y para las actitudes, la motivación y las recomendaciones de la importancia del tema en el ejercicio profesional.

La aplicación del método de proyectos de enseñanza aprendizaje en la formación universitaria aporta el desarrollo de nuevas competencias en los estudiantes, competencias que son necesarias para el desempeño profesional, principalmente en el primer trabajo, donde no se tiene mayor experiencia, como son: trabajo en equipo, investigación y el aprendizaje autónomo, planificación y toma de decisiones, liderazgo y tolerancia, solidaridad e identificación de grupo, el espíritu competitivo, etc. También se desea lograr mayores evidencias de las obtenidas hasta el momento por los investigadores del presente estudio, sobre que en el método de proyectos - MdeP destacan estudiantes que en el método tradicional mantienen un perfil bajo, porque son otras las cualidades que salen a relucir y que se deben desarrollar para ser profesionales exitosos.

Copérnico hizo girar la tierra alrededor del sol, contrario al modelo de Ptolomeo, y realizo una revolución en los cielos; el giro Copernicano en educación es: obtener mejores resultados en el aprovechamiento haciendo girar la actividad del alumno en torno a un problema (o proyecto) en vez de a la cabeza del maestro, también es una revolución: (Villegas, 2014).

\section{CONTEXTO}

A principios del año 2000, el Sistema Nacional de Adiestramiento en Trabajo Industrial - SENATI, Institución de formación profesional de nivel medio, con 12 Direcciones Zonales y 42 sedes a nivel nacional, logró la certificación ISO 9001 para la formación de técnicos industriales, para ello se documentó todos los procesos de la formación profesional; y 
con finalidad de evaluar las resultados de la formación se realizaron encuestas de opinión a los jefes de áreas de las empresas donde trabajaban, sobre los niveles de preparación de los egresados, también se realizo un trabajo sobre el grado de ocupación de los egresados.

Uno de los aportes de ambas evaluaciones fue que los estudiantes deberían ser orientados al aprendizaje autónomo y obligados a pensar, y se propuso capacitar a los docentes en el campo del aprendizaje orientado a la acción y a los proyectos; a partir de ello, el SENATI define dos métodos de enseñanza aprendizaje, el primero que se llama el método de los cuatro pasos y que siempre se ha aplicado por décadas, que incluye: la planificación, la demostración, la ejecución y la evaluación; donde los dos primeros pasos son realizados por el docente y los dos últimos por el estudiante con supervisión del docente. y el segundo, el nuevo, MdeP, orientado al aprendizaje colaborativo, autónomo y a la acción, que contempla seis pasos: informar, planificar, decidir, realizar, controlar, y valorar; en este método el docente es el facilitador y el centro del aprendizaje es el equipo de trabajo formado por cuatro a ocho estudiantes, en una competencia sana y leal por destacar; ambos métodos estaban autorizados para su ejecución.

En el año 2006, se aplican las primeras experiencias de aplicación del MdeP en la formación profesional del SENATI, en 12 sedes a nivel nacional, y los objetivos principales era desarrollar en los egresados las competencias de trabajo en equipo, aprendizaje autónomo, liderazgo, tolerancia, y toma de decisiones. Los resultados fueron los esperados; en las competencia técnicas no sufrieron disminución, los estudiantes dominaban los aspectos técnicos en conocimientos y habilidades, en forma equivalente o superior al logrado con el método de los cuatro pasos; pero con el desarrollo de las nuevas competencias antes indicadas, mostrando iniciativa, solidaridad, esfuerzo por ser los mejores, con una autoestima en crecimiento.

En el año 2008, culmina mi experiencia en el SENATI, y al final de año, pude llegar a una conclusión sorprendente, los alumnos mas destacados en los cursos de aplicación del MdeP, no son los mismos que siempre destacan en los otros cursos, el MdeP pone en relevancia y desarrolla aptitudes y capacidades que están ocultos en los métodos tradicionales de formación profesional, y que son necesarios para el desempeño laboral.

En la formación profesional universitaria, se desarrollan diversas capacidades y competencias en los estudiantes, y con el transcurrir de los años se detecto (2009 a 2012), que un aspecto importante que adolecían los estudiantes de la UNPRG, era la incursión en el mundo laboral, la gran mayoría de los estudiantes no había tenido prácticas pre profesionales en empresas, no había realizado visitas técnicas a plantas industriales, muy diferente a la temprana incursión laboral obligatoria del SENATI, y los egresados demoraban en ser empleados en las empresas relacionados con la carrera profesional que estudió y en iniciar sus investigaciones para titularse, asumiendo en un principio, aunque no se ha podido comprobar, que se debe a su poca relación con el mundo laboral y a competencias que se relacionan con asumir retos, tomar decisiones, y destacar.

En el verano del 2013 y del 2014, en el ciclo de nivelación de la UNPRG (para alumnos que desaprobaron el curso y que llevan el doble de frecuencia semanal y se hace en la mitad del tiempo que en el ciclo ordinario) aplicamos por primera vez a nivel universitario el MdeP, en el curso de circuitos de radiocomunicación de la carrera de ingeniería electrónica, con resultados mejores en el aspecto de conocimientos y habilidades, que los alumnos de los ciclos regulares, pero con el agregado de las competencias de trabajo en equipo y aprendizaje autónomo, y esperando en el futuro aplicar en un ciclo regular.

Escribano (2008), cita lo siguiente: "El ABP se define como un método de aprendizaje basado en el principio de usar problemas como punto de partida para la adquisición e integración de nuevos conocimientos, las características fundamentales son: El aprendizaje esta centrado en el alumno. El Aprendizaje se produce en pequeños grupos. Los profesores son facilitadores o guías en el proceso. Los problemas son el foco de organización y estímulo para el aprendizaje. Los problemas son un vehículo para el desarrollo de habilidades de resolución de problemas. La nueva información se adquiere a través del aprendizaje autodirigido. (Barrows, 1986)”. Sin embargo "Muchas instituciones prefieren la enseñanza con métodos tradicionales, que privilegian lo memorístico y la reproducción de saberes, sobre el descubrimiento. ..." (López, 2008). En el MdeP que realizamos, el profesor es un facilitador y un arbitro imparcial que evalúa la competencia leal entre los equipos que se forman y que tratan de lograr el mejor proyecto, tal como es en el mundo competitivo laboral.

"El Aprendizaje Basado en Proyectos (ABP) es un modelo de aprendizaje en el que los estudiantes planean, implementan y evalúan proyectos que tienen aplicación en el mundo real más allá del aula de clase (Harwell (1997)” y “Dicho aprendizaje, se produce como resultado del esfuerzo que realiza el alumno para resolver un problema o llevar a cabo un proyecto (Valero - García, 2008)” ambos citados por Saioa Villar. (Villar, 2013)

El método de proyectos aplicado en el presente estudio esta sustentado en el ABP, Aprendizaje Basado en Problemas (ABP) y Aprendizaje Basado en Proyectos (ABP) cuyas diferencias son muy finas como se observa en los planteamientos teóricos presentados, inclinándonos más por el termino proyectos porque tienen aplicación en el mundo real y la satisfacción de sus necesidades con proyectos, y a ello se ha incorporado aspectos propios de nuestra experiencia en el SENATI y la UNPRG.

En julio de 2014, se aprobó una nueva Ley Universitaria, que promueve el aprendizaje por competencias y la obligación de trabajo de investigación como requisito para obtener el grado de bachiller y otra investigación de mayor nivel para obtener el título profesional, lo que obliga a desarrollar competencias que permitan la investigación o aprendizaje autónomo y que obliguen a pensar.

Los objetivos de la aplicación del MdeP en la formación universitaria es desarrollar nuevas competencias en los estudiantes universitarios, futuros profesionales, como son el trabajo en equipo, la toma de decisiones, el liderazgo, la tolerancia, la solidaridad, en un mundo de alta competitividad en que se va ha desempeñar profesionalmente.

"La discusión en grupo cumple varios objetivos, de naturaleza intelectual, social y afectiva. El interés intelectual, como muchos autores han demostrado, se deriva fundamentalmente de que estimula a los estudiantes a explorar diversas perspectivas, resalta la complejidad de las cuestiones, les obliga a organizar su discurso y en el proceso revisar y 
quizá reformular sus ideas y ayuda a construir su sentido crítico al contrastar sus ideas con las de otros. En el ámbito social, el grupo ayuda a adoptar y reforzar hábitos democráticos y de respeto por el otro, así como a desarrollar la identidad del grupo y, de forma general, a aprender a trabajar en equipo” (Vizcarro y Juárez, 2007)

Desde el 2008 Sergio Tobón difunde los lineamientos metodológicos de la Formación Basada en Competencias en la educación superior desde el pensamiento complejo, que exige procesos de transformación curricular, la organización curricular por módulos y proyectos formativos, y la planeación del aprendizaje por problemas y talleres.(Tobón, 2008),

En la actualidad el método de aprendizaje basado en proyectos, tiene un soporte fundamental para su desarrollo en las TIC, con cada proyecto se pretende que los estudiantes hagan uso de las TIC en forma más efectiva y las utilicen para ejecutar las tareas de investigación, la escritura de informes y presentaciones electrónicas, y se realiza una evaluación auténtica, por "valoración de desempeño", (Martí, 2010), Otro avance difundido en la actualidad del aprendizaje basado en proyectos es el seguimiento de la actividad grupal de los alumnos, internet facilita reuniones no presenciales, en una experiencia de seguimiento no presencial utilizando OpenMeetings en Moodle, como en el caso de una asignatura de Ingeniería Informática. (Martí Gódia, 2013).

Los sistemas micro programables basados en plataformas de prototipos electrónicos de código abierto (open-source) basada en hardware y software flexibles y fáciles de usar, con comunicación inalámbrica como arduino, raspberry, node mcu, etc contribuyen al desarrollo de proyectos en los mas diversos campos de la vida real, facilitando en la actualidad la aplicación del aprendizaje basado en proyectos.

En Cuba el aprendizaje basado en proyectos se está aplicando en cursos de pregrado y postgrado, como en la asignatura Ecología Microbiana, en la carrera de Microbiología de la Universidad de la Habana, con el propósito de desarrollar habilidades y competencias en los estudiantes, según la Revista Universidad EAFIT de Medellín, Colombia.(Martí, 2010).

En España se esta difundiendo la innovación docente a través de Aprendizaje Basado en Proyectos y la utilización de las Tecnologías de la Información y la Comunicación en los proyectos, como es la creación de una radio educativa a través de podcast, por estudiantes del pregrado en Pedagogía de la Universidad de Burgos en España. (Ausín, 2016).

\section{DESCRIPCIÓN}

En el año 2016 en los dos ciclo ordinarios, en el curso de circuitos de radiocomunicación de ingeniería electrónica de la UNPRG, se programó la segunda mitad del ciclo (siete de diez y seis semanas) con la aplicación del MdeP, la planificación se realizo en el 2015 con el equipo formado por dos docentes, un docente de la UNPRG y un docente de Universidad Nacional Tecnológica de Lima Sur - UNTELS, se preparó el diseño curricular por proyectos, los planes de sesión, los formatos de evaluación y las rubricas.

"El método de proyectos de enseñanza aprendizaje, permite desarrollar el modelo denominado de “acción completa”, este modelo consta de seis pasos que son los siguientes: Informar, Planificar, decidir, realizar, controlar y valorar.” (SENATI,
2007), y previo al inicio se realiza una etapa de sensibilización, necesaria cuando el grupo no ha tenido experiencia anterior sobre el usos del método de proyectos, aprendizaje basado en problemas o proyectos o similares.

\section{A.- Sensibilización}

\section{La sensibilización tiene tres etapas}

1.- Actividad grupal: Se prepara con anticipación el ambiente en el que se desarrollara la actividad grupal, aula o laboratorio con mesas donde puedan trabajar con materiales como cartulina, cartón, diferentes tipos de papeles, pegamento lento y rápido, lápiz, reglas, tijeras, engrampador, chinches. papel de pegar, etc.

Se conforman los equipos de trabajo con cuatro a ocho miembros, tratando de tener equipos de igual cantidad de miembros o algunos con un miembro mas; y se le comunica los materiales que cada grupo debe traer para la sesión de sensibilización. Las actividades pueden ser diversas, que ayuden a comprender la necesidad de trabajar en equipo, tomar decisiones, asumir el liderazgo, tener tolerancia, solidaridad y ayuda mutua. La actividad grupal se realizo en una sesión de 3 horas académicas de 50 minutos, las otras dos en otra sesión.

En la sensibilización del 2016-1, se uso la construcción de una torre, donde se dio una hoja con breves instrucciones: "METODO DE PROYECTOS DE ENSEÑANZA APRENDIZAJE, El Proyecto de la Torre, con el material requerido para la sesión, construir una torre que sea tan bonita, tan alta y tan estable como el equipo pueda lograr en una hora quince minutos, la torre debe tener estabilidad sin apoyo adicional y externo". Se evaluó los resultados en los tres aspectos indicados: altura, belleza y estabilidad, elaborando un cuadro de evaluación en la pizarra y se presento el orden de méritos de los equipos que participaron.

En la sensibilización del 2016-2, se uso la técnica de creatividad 635 de Warfield (6 personas, 3 ideas, en 5 minutos) para generar ideas sobre problemas y proyectos para solucionarlos y la técnica de los 6 sombreros para pensar de Edward de Bono (blanco, rojo, amarillo, verde, azul y negro) para hacer reflexionar a los otros equipos, desde diferentes puntos de vistas, sobre sus propuestas.

2.- Sustentación de la experiencia: En la siguiente sesión cada equipo sustenta el informe preparado sobre de la experiencia de la actividad grupal y el desempeño de cada miembro del equipo en esta actividad, contando con el apoyo de imágenes y videos, relatando los pasos y resultado final de la construcción de la torre en el primer caso y sobre las aplicaciones de las técnicas 635 y 6 sombreros para la creatividad en la solución de los problemas, en el segundo caso.

3.- Explicación del método de proyectos: Después de consolidar las experiencias de la actividad grupal, el docente explica los objetivos del MdeP y las competencias que ayuda a desarrollar, las fases del método, en que consiste cada una y las obligaciones de los equipos en cada fase; las metas que se desea alcanzar al finalizar las siete semanas que se programó para llevar a cabo la aplicación del método de proyectos en el curso de circuitos de radiocomunicación, en cada semana se tiene dos sesiones, cada una de 150 minutos. La sensibilización se realizo en la novena semana del ciclo. 


\section{B.- Informar}

La primera fase del MdeP es Informar, en ella, el docente entrega la información general del proyecto, como son: el tema en que se enmarca el proyecto, la información que deben preparar en al primera parte del trabajo para complementar la información general y hacer más especifico el tema del proyecto a desarrollar, recopilan y analizan de modo autónomo información adicional que estiman será necesaria para las fases de planificación y ejecución del proyecto.

A nivel del SENATI, se les proporcionaba preguntas guía para la búsqueda de la información necesaria y suficiente, pero a nivel de la UNPRG, se les hace sugerencias y se les pide que ellos elaboren un conjunto de interrogantes propias, equivalentes a las preguntas guía y busquen las respuestas y alternativas de solución a problema de desean solucionar y que este enmarcado en el tema. En la aplicación del MdeP en el SENATI, el acceso a la información tenia sus limitaciones, se les proporcionaba manuales, fascículos, libros y una computadora con internet, que tenían que compartir todos los equipos, con un tiempo aproximado de treinta minutos cada equipo; en el caso de la UNPRG, la información esta disponible en todos los aspectos, impresos, virtuales, en línea, etc. además cada equipo cuenta con tres o más laptops de propiedad de los estudiantes y cuentan con internet. La fase Informar se realizo en las dos sesiones de la decima semana, en la última sesión se realizó una plenaria en que cada equipo explico las preguntas que se plantearon, las respuestas que obtuvieron y la pertinencia de las preguntas con el proyecto específico que piensan desarrollar y que se enmarca en el tema general planteado.

\section{C.- Planificar}

Los estudiantes tienen que planificar el desarrollo del proyecto y buscar información adicional de acuerdo a sus necesidades. Cada equipo elabora de modo autónomo un plan de trabajo completo, que debe tener definido todo lo referente a la construcción del proyecto y además debe incluir la organización del equipo y las responsabilidades de cada miembro. Esta fase se realizo en la semana decima primera, igualmente en la última se realizo una plenaria, donde cada equipo sustento su plan de trabajo y recibió las observaciones y aportes de los otros equipos y del docente en su papel de facilitador.

En esta fase se identifica las similitudes de los proyectos y de las alternativas de solución, el facilitador explica que aspectos deben definir los equipos para diferenciarse en la ejecución de los proyectos.

\section{D.- Decidir}

Los equipos, teniendo en cuenta las observaciones y aportes recibidos en la sesión anterior, realizan las mejoras y modificaciones que estiman conveniente, y toman las decisiones para la versión final de su plan de trabajo. En la segunda sesión se realiza una plenaria donde cada equipo entrega su plan de trabajo impreso e informa los detalles de su plan de trabajo. Se aprueba el plan y su compromiso con el colectivo del curso. Esta fase se realizo en la décima segunda semana.

\section{E.- Ejecutar}

Los equipos ejecutan las actividades necesaria para alcanzar su meta, que es construir el proyecto con las características definidas por ellos y cumplir con su plan de trabajo. El docente observa el avance de los trabajos y solo interviene en caso de inminente peligro para los equipos o personas, puede orientar en forma general, para todos, ante el requerimiento de un equipo, pero no puede dar una solución especifica para cada caso, el docente es un arbitro entre los equipos que desarrollan su proyecto como un reto y una competencia leal. Se realizó en la décima tercera semana.

\section{F.- Controlar}

Esta fase tiene una parte a cargo de los mismos miembros del equipo, y se lleva a cabo en forma conjunta con la fase ejecutar, se trata de verificar el cumplimiento de las características y especificaciones técnicas de cada parte de los circuitos que forman parte del proyecto. Otra parte esta a cargo del docente, quien verifica el desempeño de cada miembro del equipo y se lleva a cabo en forma continua en la fase ejecutar, y monitorea el resultado de los avances de las diferentes partes del proyecto. En la segunda sesión de la décima cuarta semana se realiza la demostración del funcionamiento del proyecto evaluando los siguientes aspectos: Proceso de ejecución, Orden y seguridad, Precisión y acabado, Funcionamiento, y Tiempo de ejecución; cada aspecto un 20\%. Esta fase se realizó en la décima cuarta semana. Los Equipos pueden hacer la demostración en la primera sesión si tienen el proyecto terminado. Los equipos que se retrasan, presentan el estado de avance del proyecto, y pueden hacer su demostración en la siguiente sesión, afectando el proceso y tiempo de ejecución.

\section{G.- Valorar}

En la primera sesión de la décima quinta semana los equipos presentan su informe de ejecución del proyecto y sustentan el informe destacando las características más importantes del producto final del proyecto. Completan los formatos de autoevaluación y coevaluación que forman parte del diseño de aplicación del MdeP.

En la segunda sesión de esta última semana, los equipos presentan y exponen la valoración del trabajo realizado compartiendo las experiencias, vivencias y aprendizaje logrado con el método de proyectos, la utilidad que pueda tener en el futuro para su desempeño profesional, y el docente presenta su informe respecto a los resultados de la aplicación del MdeP en el curso de circuitos de radiocomunicación, informando el orden de mérito por equipo y los resultados de la autoevaluación y coevaluación en el desempeño y contribución de cada miembro al funcionamiento del equipo y al logro de la meta propuesta.

\section{Resultados}

\section{A. Ciclo 2016-1}

En el ciclo 2016-I, la información general fue: Tema: Modulación en amplitud y en frecuencia, y la Competencia: El ingeniero electrónico diseña, construye y prueba el funcionamiento de un prototipo de transmisor de amplitud modulada y de frecuencia modulada, de baja potencia y de alta calidad. Participaron 20 estudiantes, formándose cinco equipos de cuatro estudiantes cada equipo.

En general el aprendizaje de los estudiantes ha sido claramente mas productivo y ordenado con el MdeP, que con el método tradicional, de impartir clases con ponencias 
didácticas para la teoría, practicas de laboratorio para las habilidades y motivación y recomendaciones para las actitudes, con el MdeP se tiene una actividad integral a cargo de los propios alumnos, la investigación y consolidación de los conocimientos, la planificación, decisión y control para la construcción práctica del prototipo como aplicación de conocimiento, y el desarrollo de competencias del tipo personal social necesarias para el ejercicio laboral, como son el liderazgo, el trabajo en equipo, la toma de decisiones, etc.

El orden de mérito final de la aplicación del método de proyectos en el curso de circuitos de radiocomunicación fue el que se presenta en la tabla siguiente:

Tabla 1 Resultados MP ciclo 2016 -1

\begin{tabular}{|c|c|l|l|}
\hline ORD. & EQ & PROYECTOS CICLO 2016 1 & CALIDAD \\
\hline $\mathbf{1}$ & A & $\begin{array}{l}\text { TRANSMISOR DE FM CON } \\
\text { UN TRANSISTOR }\end{array}$ & $\begin{array}{l}\text { MUY } \\
\text { BUENA }\end{array}$ \\
\hline $\mathbf{2}$ & D & $\begin{array}{l}\text { TRANSMISOR DE AM CON } \\
\text { TRANSISTORES }\end{array}$ & BUENA \\
\hline $\mathbf{3}$ & B & $\begin{array}{l}\text { TRANSMISOR DE FM CON } \\
\text { DOS TRANSISTOR }\end{array}$ & BUENA \\
\hline $\mathbf{4}$ & C & $\begin{array}{l}\text { TRANSMISOR DE AM CON } \\
\text { TRANSISTORES }\end{array}$ & REGULAR \\
\hline $\mathbf{5}$ & E & $\begin{array}{l}\text { TRANSMISOR DE FM CON } \\
\text { UN TRANSISTOR }\end{array}$ & REGULAR \\
\hline
\end{tabular}

Evidenciados en los proyectos de muy buena calidad que se demostraron y sustentaron los equipos, destacando el trabajo del equipo A como se muestra en las imágenes de las Figura 1, por su calidad y alcance, seguido por los equipos D y B con buena calidad y mediano alcance y finalmente los equipos $\mathrm{C}$ y $\mathrm{E}$, como se muestra en la Tabla 1.

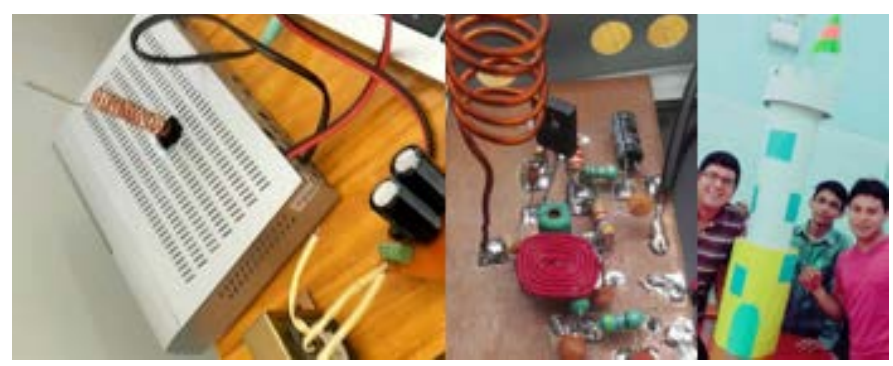

Figura 1. Evidencias del equipo de trabajo A, con su transmisor FM, y su torre en la sensibilización.

\section{B. Ciclo 2016-1I}

En el ciclo 2016-II, la información general fue: Tema: Comunicación inalámbrica en radio frecuencia - RF, y la Competencia: El ingeniero electrónico diseña, construye y prueba el funcionamiento de un prototipo de módulo de comunicación inalámbrica en frecuencias de radio y de alta calidad. Participaron 28 estudiantes, formándose cinco equipos, tres de seis estudiantes y dos de cinco estudiantes.

El orden de mérito final de la aplicación del método de proyectos en el curso de circuitos de radiocomunicación fue el que se presenta en el cuadro siguiente:

Tabla 2 Resultados MP ciclo 2016-2

\begin{tabular}{|c|c|l|l|}
\hline ORD. & EQ. & PROYECTOS CICLO 2016 2 & CALIDAD \\
\hline $\mathbf{1}$ & B & $\begin{array}{l}\text { COMUNICACIÓN CON UN } \\
\text { SEGUIDOR DE LINEA }\end{array}$ & $\begin{array}{l}\text { MUY } \\
\text { BUENA }\end{array}$ \\
\hline $\mathbf{2}$ & D & $\begin{array}{l}\text { ENTORNO DOMOTICO CON } \\
\text { INTERNET DE LAS COSAS }\end{array}$ & $\begin{array}{l}\text { MUY } \\
\text { BUENA }\end{array}$ \\
\hline $\mathbf{3}$ & A & $\begin{array}{l}\text { APLICACIÓN DE DOS } \\
\text { TRANSCEPTORES DE RF }\end{array}$ & BUENA \\
\hline $\mathbf{4}$ & C & $\begin{array}{l}\text { COMUNICACIÓN RF PARA } \\
\text { UNA CASA DOMOTICA }\end{array}$ & BUENA \\
\hline $\mathbf{5}$ & E & $\begin{array}{l}\text { CONTROL AMBIENTAL } \\
\text { CON NODE MCU }\end{array}$ & BUENA \\
\hline
\end{tabular}

En la imagen de la figura No. 2 se observa la pista, el carrito seguidor de línea y el equipo móvil que se comunica con el carrito para saber los detalles del funcionamiento de mediante reporte de datos e iconos.

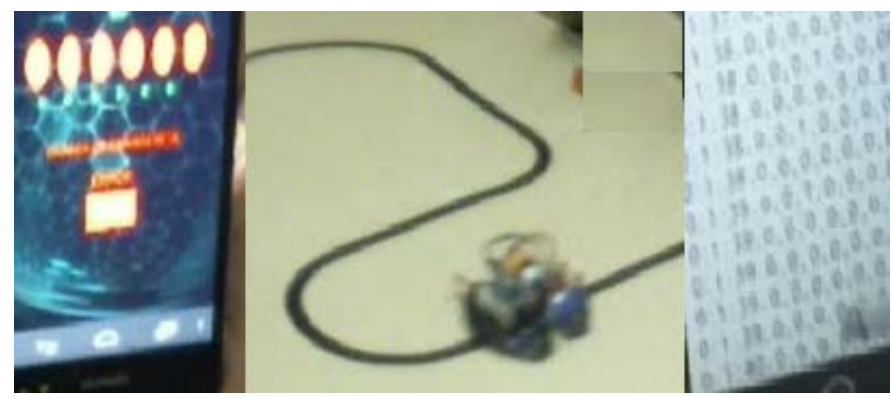

Figura 2. Evidencias del equipo de trabajo B, con su carrito seguidor de línea y su información en el móvil

\section{Comparación de notas}

En los cuadros se muestran las comparaciones del promedio de notas por equipos de trabajo, teniendo la nota del curso de circuitos de radiocomunicación - CIRACO, en el que se ha aplicado el MdeP y las notas del curso anterior, circuitos electrónicos 3 - CIRELO3, que se llevo a cabo con el método tradicional de enseñanza aprendizaje. La escala de notas es vigesimal ( de 0 a 20).

Tabla 3 Orden de merito por equipos ciclo 2016-1

\begin{tabular}{|c|c|c|c|c|}
\hline \multicolumn{5}{|c|}{ CICLO 2016 -1 } \\
\hline & \multicolumn{2}{|c|}{ PROMEDIOS } & \multicolumn{2}{c|}{ ORDEN DE MERITO } \\
\hline EQUIPO & CIRACO & CIRELO3 & CIRACO & CIRELO3 \\
\hline A & 16.25 & 14.00 & 1 & 1 \\
\hline B & 14.25 & 11.50 & 3 & 5 \\
\hline C & 12.75 & 12.00 & 4 & 3 \\
\hline D & 14.75 & 13.25 & 2 & 2 \\
\hline E & 12.50 & 11.75 & 5 & 4 \\
\hline
\end{tabular}

Tabla 4 Orden de mérito por equipos, ciclo 2016-2

\begin{tabular}{|c|c|c|c|c|}
\hline \multicolumn{5}{|c|}{ CICLO 2016 -2 } \\
\hline & \multicolumn{2}{|c|}{ PROMEDIOS } & ORDEN DE MERITO \\
\hline EQUIPO & CIRACO & CIRELO3 & CIRACO & CIRELO3 \\
\hline A & 14.17 & 11.83 & 3 & 5 \\
\hline B & 14.50 & 12.17 & 1 & 3 \\
\hline C & 13.83 & 12.00 & 4 & 4 \\
\hline D & 14.20 & 12.60 & 2 & 1 \\
\hline E & 13.40 & 12.40 & 5 & 2 \\
\hline
\end{tabular}

En los cuadros se observa que solo tres equipos de diez equipos mantiene su orden de mérito, A y D en el 2016-1 y C en el 2016-2, en el curso de circuitos de radiocomunicación CIRACO en relación con el curso de Circuitos Electrónicos 3 - CIRELO3, Los demás equipos cambian su orden de merito, lo que indica que hay un cambio en el rendimientos de 7 
equipos de los 10 que participaron, 30\% mantiene y el $70 \%$ cambia, esto se puede deber a que el MdeP explota otras características de los alumnos, y por supuesto desarrolla otras competencias distintas a las que realiza el método tradicional.

En la tabla No. 5, se tienen los promedios de las notas de los alumnos en cada curso, por semestre y también la relación que tienen las notas de los alumnos con el promedio; formando cuatro grupos de alumnos: Primer Grupo.- alumnos con notas mayores al promedio en ambos curso (30\% y 25\%), Segundo Grupo.- alumnos con notas mayores que el promedio en CIRACO (MdeP) y menores en CIRELO3 (25\% y 36\%), Tercer Grupo.- alumnos con notas menores que el promedio en CIRACO (MdeP) y mayores en CIRELO3 (15\% y 14\%) y el Cuarto Grupo.- alumnos con notas menores que el promedio en ambos cursos (30\% y 25\%).

Se puede observar, analizando el Segundo Grupo y el Tercer Grupo, que en el 2016-1 el 40\% (25\% + 15\%) y en el 2016-2 el 50\% (36\% + 14\%) de los alumnos tiene un rendimiento distinto cuando se aplica el método tradicional y el Método de Proyectos - MdeP.

Tabla 5 Promedios y su relación con grupos de notas

\begin{tabular}{|l|c|c|c|c|}
\hline \multicolumn{1}{|c|}{ RUBRO } & $\mathbf{2 0 1 6 - 1}$ & $\mathbf{\%}$ & $\mathbf{2 0 1 6 - 2}$ & $\boldsymbol{\%}$ \\
\hline PROMEDIO CIRACO & 14.1 & & 14.0 & \\
\hline PROMEDIO CIRELO3 & 12.5 & & 12.2 & \\
\hline CIRACO Y CIRELO3 MAYORES & 6 & 30 & 7 & 25 \\
\hline CIRACO MAYOR CIRELO3 MEN & 5 & 25 & 10 & 36 \\
\hline CIRACO MENOR CIRELO3 MAY & 3 & 15 & 4 & 14 \\
\hline CIRACO Y CIRELO3 MENORES & 6 & 30 & 7 & 25 \\
\hline TOTAL & 20 & 100 & 28 & 100 \\
\hline
\end{tabular}

\section{CONCLUSIONES}

1.- El método de proyectos - MdeP en la formación profesional universitaria aprovecha y desarrolla cualidades personales que son pasadas por alto en los métodos tradicionales de ponencias didácticas y prácticas de laboratorio, en el método de proyectos se realiza la investigación para adquirir los conocimiento, la planificación, la decisión y el control por el equipo de trabajo, su aprendizaje es autónomo. Y el resultado en conocimientos, habilidades y actitudes son mejores evidenciados en los trabajos resultado de los proyectos ejecutados.

2.- El método de proyecto - MdeP desarrolla competencias generales como el trabajo en equipo, la toma de decisiones, el liderazgo y la tolerancia, la solidaridad, investigación y aprendizaje autónomo, muy necesarios en el ejercicio profesional, principalmente en los primeros años, cuando el nuevo profesional no tiene la experiencia. Se evidencia en el desempeño y los resultados obtenidos con autonomía.

3.- Estamos en la comprobación, cada vez con mayores evidencias, que los alumnos que suelen tener un perfil bajo en el proceso de enseñanza aprendizaje tradicional, destacan cuando se aplica el método de proyectos - MdeP. Entre el $40 \%$ y el $50 \%$ de los alumnos cambia de tener notas menores que el promedio con el método tradicional a tener notas mayores que el promedio y viceversa.

\section{AGRADECIMIENTOS}

Nuestro agradecimiento los docentes del SENATI, UNPRG y UNTELS por los diálogos, intercambio de opiniones y comentarios para mejorar la aplicación del método de proyectos, y nuestro reconocimiento a los alumnos que llevaron el curso de circuitos de radiocomunicación de ingeniería electrónica de la UNPRG en los dos ciclos del año 2016, por su excelente participación en las experiencias.

\section{REFERENCIAS}

Ausín, V. Abella, V. Delgado, V. Hortigueda D. (2016) Aprendizaje basado en proyectos a través de las TIC. Una experiencia de innovación docente desde las aulas universitarias, Formación Universitaria, Vol. 9(3), pp 3138, 2016, Burgos - España.

Escribano, A. y Del Valle, A. (2008). El Aprendizaje Basado en Problemas - Una propuesta metodológica en Educación Superior, Narcea SA de ediciones, Madrid España.

López, M. (2008). El aprendizaje basado en problemas una propuesta en el contexto de la educación superior en México, tiempo de educar, vol. 9, núm. 18, juliodiciembre 2008 pp. 199-232.

Martí, J. Heydrich, M. Rojas, M. Hernández, A. (2010) Aprendizaje basado en proyectos: una experiencia de innovación docentes, Revista Universidad EAFIT, Vol. 46 No. 158-2010, pp 11-21, Medellín - Colombia

Martí Gódia, E. Gurguí, A. Hernández, A. Gil, D (2013) ABP Semipresencial: una propuesta de ABP usando herramientas online. Organización, seguimiento y evaluación. Educar para Transformar, Barcelona España.

SENATI (2007) Método de proyectos de enseñanza aprendizaje, Fascículo de Aprendizaje de 25 paginas.

Tobón, S. (2008).Formación basada en competencias en la educación superior - El enfoque complejo, Curso Iglú 2008, Guadalajara- México.

Villar, S. (2013). Aprendizaje Basado en Proyectos - Facultad de Educación, Universidad de Zaragoza, Zaragoza España.

Villegas, J. (2014), El ABP Rediseñado - Una versión personal del Aprendizaje Basado en Problemas, libro electrónico ISBN 978-1-4633-7679-6.

Vizcarro C. y Juárez E. (2007) ¿Qué es y como funciona el aprendizaje en problemas? Cap. 1 del libro Metodología del aprendizaje basado en problemas. 